\title{
Capillary bridges between a plane and a cylindrical wall
}

\author{
Etienne Reyssat ${ }^{1} \dagger$, \\ ${ }^{1}$ PMMH, CNRS UMR 7636 - ESPCI - UPMC Université Paris 6 - UPD Université Paris 7, \\ 10 rue Vauquelin, 75005 Paris, France, EU
}

(Received ?; revised ?; accepted ?. - To be entered by editorial office)

We report experimental, theoretical and numerical results on the shapes of liquid menisci connecting a planar boundary and the surface of a horizontal cylinder placed above. The gradient of confinement traps the wetting drops in the most confined regions, which promotes their elongation along the line of smallest gap between the walls. The experimental shapes of these stretched capillary bridges are shown to be in good quantitative agreement with the numerical solution of the equation describing their contour. In particular, we show that the measured shapes are better described when taking into account the correction resulting from the coupling of in-plane and transverse interfacial curvatures calculated by Park \& Homsy (J. Fluid Mech., vol. 139, 1984, pp. 291-308) over thirty years ago.

Key words: drops and bubbles, interfacial flows (free surface), liquid bridge

\section{Introduction}

Over thirty years ago, in a highly cited paper in the Journal of Fluid Mechanics, Park \& Homsy (1984) derived the pressure jump across the moving interface between two fluids confined in a Hele-Shaw cell using double asymptotic methods. This result has since been widely applied in the description of moving menisci in confined media. Relevant phenomena include various viscous fingering situations (Bensimon et al. 1986; Geoffroy et al. 2006), the Kelvin-Helmholtz instability (Gondret \& Rabaud 1997; Plouraboué \& Hinch 2002) or the printer's instability between journal bearings (Rabaud et al. 1991; Rabaud 1994). Using simpler techniques, Paterson (1996) obtained the static version of the result of Park \& Homsy (1984), as well as a first experimental check by analyzing the shapes of menisci distorted by wetting defects in Hele-Shaw cells (Paterson et al. 1995).

A huge number of papers in the literature describe the shapes of menisci confined between particles and the forces they exert. The importance of these small confined liquid drops is obvious when building sand castles at the beach, as the strong cohesion of the grains is caused by the minute amounts of liquid between the grains. The forces exerted by capillary bridges often dominate the cohesion and mechanical behavior of powders, wet soils and granular materials in general (Mitarai \& Nori 2006). They strongly affect the adhesion or friction between solid particles or surfaces and consequently have a huge practical impact in many small-scale applications (Butt \& Kappl 2009). The precision of dip-pen nanolithography techniques, for instance, depends on the static and dynamic properties of capillary bridges between an atomic force microscope tip and the printed surface (Eichelsdoerfer et al. 2014). The presence of liquid interfaces during the

$\dagger$ Email address for correspondence: etienne.reyssat@espci.fr 
fabrication process of microstructures can lead to their collapse and ruin (Roman \& Bico 2010). The adhesion of some insects relies partially on the presence and control of capillary bridges formed by a viscous secretion between their leg pads and environing surfaces (Federle et al. 2002). In all of these applications, the static forces and shapes of liquid menisci are crucial. Their dynamic properties also often play a dominant role in the macroscopic mechanical behavior of materials or animals.

In this paper, we start by giving a simple derivation of the outcome of the work by Park \& Homsy (1984) in the particular static case. Building on this result, we compute higher-order terms in the development of $\Delta P$ in terms of $h / R$, where $2 h$ is the thickness of the Hele-Shaw cell and $R$ is the interface radius of curvature in the plane of the confining walls. We then use the first-order approximation to determine the shapes of capillary bridges connecting a plane wall and a cylindrical boundary. We compare analytical predictions and numerical solutions of the model with experimentally measured shapes.

\section{Pressure drop across a static interface in a Hele-Shaw geometry}

We consider a circular puck of liquid bridging the parallel plates of a Hele-Shaw cell, as shown in figure 1a and assume that the liquid is totally wetting the walls. As discussed, for instance, by Ajaev \& Homsy (2006), de Gennes (1985) and Bonn et al. (2009), this means that the air/liquid interface of the drop connects to a liquid film adsorbed on the plates around the drop. The thickness of this layer is usually up to $100 \mathrm{~nm}$, at least three orders of magnitude smaller than the wall separation of the Hele-Shaw cells considered here. The region where the air/liquid interface connects to this microscopic film is commonly called the "apparent" contact line, and will in the following be considered as the boundary of the capillary bridges we consider.

The capillary pressure inside the drop is given by the Laplace law (de Gennes et al. 2004):

$$
\Delta P=\gamma\left(\frac{1}{R_{1}}+\frac{1}{R_{2}}\right)
$$

where $\gamma$ is the surface tension of the liquid, and $R_{1}$ and $R_{2}$ are the principal radii of curvature of the liquid/air interface. The two natural scales for curvature in this problem are the thickness $2 h$ of the Hele-Shaw cell, and the radius of curvature $R=1 / \mathcal{C}$ of the interface in the plane of the plates, measured at mid-height between the walls $(z=0)$. While it may be thought that $R$ and $h$ are the principal radii of curvature of the interface, both quantities are actually coupled and can generally not be identified with $R_{1,2}$.

We now consider the mechanical equilibrium of the half of the drop located in the region $y>0$ of space, as shown in figure $1 \mathrm{~b}$. We estimate the projection along the $y$ direction of pressure and capillary forces applied on this semidrop. The pressure difference $\Delta P$ between the inside and outside of the drop applies on the surface area $\Sigma$ of the drop cross-section, represented as the grey area in figures 1a-b. At first order in $h / R$, $\Sigma \simeq 4(R+h) h-\pi h^{2}$. The pressure force acting along $O y$ is thus

$$
F_{P}=4 h R \Delta P\left(1+\left(1-\frac{\pi}{4}\right) \frac{h}{R}+O\left(\left(\frac{h}{R}\right)^{2}\right)\right)
$$

Surface tension acts at the boundaries of the semidrop, and counterbalances pressure forces to ensure equilibrium. The projected length of the lines normal to $O y$ involves two terms. The first arises from the lines where the liquid/air interface reaches the upper and lower plates, which are semicircles of radius $R+h$ at first order in $h / R$. At these 
(a)

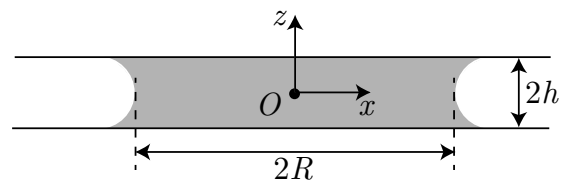

(b)

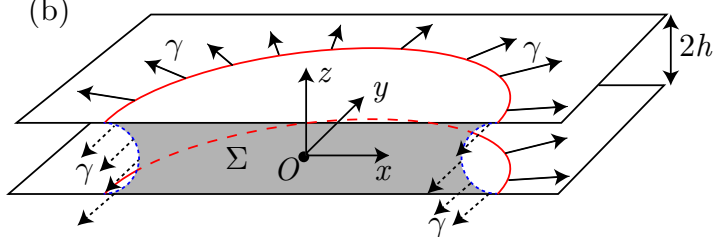

(c)

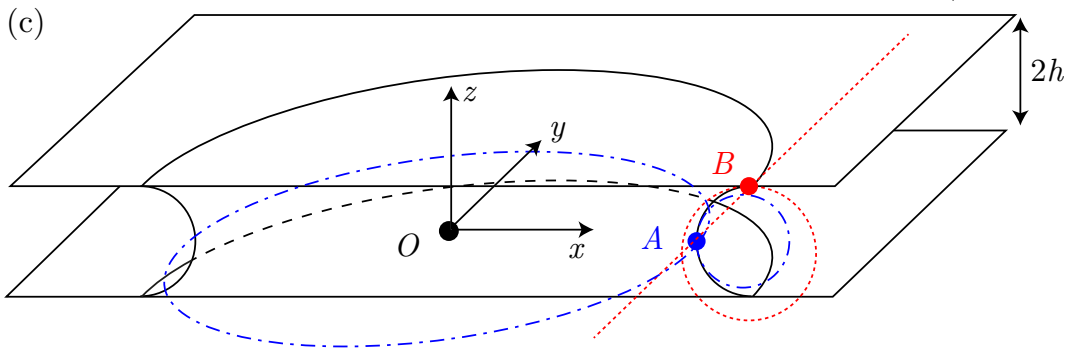

FIGURE 1. (a) Side view of a circular liquid drop of equatorial radius $R$ bridging to parallel glass plates separated by a gap of thickness $2 h . O$ is the center of the drop. (b) We consider the mechanical equilibrium of the half of the drop located in the $y>0$ half-space. The pressure difference $\Delta P$ applies on the surface area $\Sigma$ of the drop cross-section, the light grey area on the sketch. Surface tension forces pull on the bounding lines with an intensity $\gamma$ per unit length. Contributions from the apparent contact lines are sketched using full arrows, while forces associated to lateral menisci are shown as dotted arrows. (c) Cross-section of the drop through a vertical symmetry plane. The osculating circles are sketched to show the principal radii of curvature of the interface at a point $A$ along the equator of the drop (dash-dotted lines) and $B$ at the apparent contact line (dotted lines).

apparent contact lines, surface tension pulls on the drop radially, as shown by figure $1 \mathrm{~b}$. The length of these lines is $2 \times \pi(R+h)$, and their projected length along $O x$ is $4(R+h)$. The corresponding capillary force thus pulls the drop towards the $y>0$ region with intensity $4 \gamma(R+h)$. The other place where interfacial forces apply is along the menisci on the sides of the semidrop, the dotted lines in figure $1 \mathrm{~b}$, of length $2 \times \pi h$. Capillary forces act here towards $y<0$ with intensity $2 \pi \gamma h$. The total capillary force acting on one half of the drop is thus

$$
F_{\gamma} \simeq \gamma(4 R+(4-2 \pi) h)
$$

or more precisely

$$
F_{\gamma}=4 R \gamma\left(1+\left(1-\frac{\pi}{2}\right) \frac{h}{R}+O\left(\left(\frac{h}{R}\right)^{2}\right)\right)
$$

At mechanical equilibrium, pressure and capillary forces exactly balance: $F_{P}+F_{\gamma}=0$. Keeping terms of order up to $h / R$ then yields the expected result:

$$
\Delta P=-\frac{\gamma}{h}\left(1-\frac{\pi}{4} \frac{h}{R}+O\left(\left(\frac{h}{R}\right)^{2}\right)\right)
$$




\section{Comments}

\section{Energy}

The pressure difference across the drop interface can also be derived using energy considerations. Let us first express the volume $V$ of a drop as a function of $h$ and $R$ :

$$
V=2 \pi R^{2} h\left(1+(2-\pi) \frac{h}{R}++O\left(\left(\frac{h}{R}\right)^{2}\right)\right)
$$

An infinitesimal change $\mathrm{d} V$ in the drop volume corresponds to an increase of the drop radius $\mathrm{d} R$. The work done by pressure forces during this transform is

$$
\delta W=-\Delta P \mathrm{~d} V=-\Delta P 4 \pi R h\left(1+\left(1-\frac{\pi}{4}\right) \frac{h}{R}++O\left(\left(\frac{h}{R}\right)^{2}\right)\right) \mathrm{d} R
$$

The work of pressure forces is converted into surface energy, which arises through two contributions. First, the wetting film around the drop gets partially covered as $R$ increases, thus reducing the area of the liquid/air interface. At first order in $h / R$, the surface area $\Sigma_{f}$ of the film covered by the drop is

$$
\Sigma_{f}=2 \times \pi R^{2}\left(1+\frac{h}{R}++O\left(\left(\frac{h}{R}\right)^{2}\right)\right)^{2}
$$

The capillary energy variation corresponding to an increase of $\Sigma_{f}$ is

$$
\mathrm{d} E_{\gamma_{f}}=-\gamma \mathrm{d} \Sigma_{f}=-\gamma 4 \pi R\left(1+\frac{h}{R}+O\left(\left(\frac{h}{R}\right)^{2}\right)\right) \mathrm{d} R
$$

The lateral surface of the drop, of area $\Sigma_{l} \simeq 2 \pi^{2} R h$, increases as the drop is inflated. The related energy change is

$$
\mathrm{d} E_{\gamma_{l}}=\gamma \mathrm{d} \Sigma_{l}=\gamma 2 \pi^{2} h\left(1+O\left(\frac{h}{R}\right)\right) \mathrm{d} R
$$

Equating the pressure work and the surface energy change $\left(\delta W=\mathrm{d} E_{\gamma_{f}}+\mathrm{d} E_{\gamma_{l}}\right)$ also leads to equation 2.5 .

\section{Interfacial principal curvatures}

The shape of a static drop confined between parallel walls is thus that of a Delaunay surface (Delaunay 1841), i.e. a surface of revolution with constant mean curvature $\kappa$ :

$$
\kappa=\frac{1}{h}-\frac{\pi}{4 R}
$$

It may be thought that the curvature of the interface in the plane normal to the plates is $1 / h$ whereas the curvature in the plane parallel to the plates should be $1 / R$. However, as suggested by Nagel et al. (2014), the two contributions do not necessarily reflect the principal radii of curvature of the interface, and equation 2.11 may be rearranged to better highlight the principal curvatures at different locations on the interface. The intersection of the liquid/air interface with the horizontal mid-height plane $O x y$ is a perfect circle of radius $R$. At a point $A$ located on this circle, as defined in figure $1 \mathrm{c}$, the principal radius of curvature of the interface in the $A x y$ plane parallel to the plates is indeed $R_{1 A}=-R$. To satisfy equation 2.11, the transverse curvature is thus $1 / R_{2 A}=1 / h+(1-\pi / 4) / R$, where $R_{2 A}<h$. At point $B$, the interface is not curved in the transverse (Byz) plane: $R_{1 B}=\infty$. The curvature in the $B x z$ plane is $1 / R_{2 B}=\kappa=1 / h-\pi / 4 R$, with $R_{2 B}>h$. 
In the special case of a very large drop $(R / h \rightarrow+\infty)$, the in-plane and transverse curvatures decouple. Then, the intersection of the drop boundary with a vertical plane is exactly a semicircle of radius $h$, and the pressure in the drop is

$$
\Delta P=-\frac{\gamma}{h}
$$

The case of partial wetting

Using a similar force or energy balance, one can derive the pressure jump in the case of a partially wetting fluid. We call $\theta$ the contact angle of the fluid on the solid walls. In this case, the pressure jump is

$$
\Delta P=-\frac{2 \gamma}{h} \cos \theta\left(1-\frac{h}{2 R \cos \theta}\left(\frac{\sin \theta}{2}+\frac{1}{2 \cos \theta}\left(\frac{\pi}{2}-\theta\right)\right)+O\left(\left(\frac{h}{R}\right)^{2}\right)\right)
$$

This equation is in agreement with the interpretation in terms of line tension given in Paterson et al. (1995) and Paterson (1996). The limit of this result in the case of total wetting $(\theta=0)$ is indeed equation 2.5. In the special case $\theta=\pi / 2$, the liquid interface is a cylinder of radius $R$ and height $2 h$ and equation 2.13 reduces to

$$
\Delta P=\frac{\gamma}{R}
$$

\section{Higher-order corrections}

Equation 2.5 remains a good approximation of the interfacial curvature as long as $h / R$ is small. If the bounding plates separate ( $h$ increases), $h / R$ becomes of order one, and the bridge ruptures at some critical wall separation. In this regime, the first-order development in the pressure (or curvature) most likely fails, and one may need to compute higher-order terms. As shown by Melrose (1966), it is possible to express analytically the shape of the meniscus using elliptic integrals. Building on the result of Melrose (1966), one can develop the constant curvature $\kappa$ of the meniscus as a power series in $h / R$. This is done through an iterative process, and yields the first terms of the development:

$$
\begin{aligned}
\kappa= & \frac{1}{h}\left[1-\frac{\pi}{4} \frac{h}{R}+\left(\frac{\pi}{4}-\frac{\pi^{2}}{32}\right)\left(\frac{h}{R}\right)^{2}-\left(\frac{9 \pi}{32}-\frac{\pi^{2}}{8}+\frac{5 \pi^{3}}{512}\right)\left(\frac{h}{R}\right)^{3}\right. \\
& \left.+\left(\frac{11 \pi}{32}-\frac{21 \pi^{2}}{64}+\frac{35 \pi^{3}}{512}-\frac{\pi^{4}}{256}\right)\left(\frac{h}{R}\right)^{4}+O\left(\left(\frac{h}{R}\right)^{5}\right)\right]
\end{aligned}
$$

The appendix at the end of the paper gives the main steps to compute the curvature at an arbitrary order in $h / R$.

\section{Shape of a drop between a plane and a horizontal cylinder}

\subsection{Experiments}

We now apply the result of equation 2.11 to derive the shape of a liquid volume connecting a horizontal plane and a cylinder. Such elongated capillary bridges may in practice be found between journal bearings (Rabaud et al. 1991; Rabaud 1994) or along small worms crawling on moist surfaces (Sauvage et al. 2011). The experimental setup is shown in figure $2 \mathrm{a}, \mathrm{b}$ and $\mathrm{c}$. We introduce a drop of silicone oil between a horizontal glass plate and a surmounting cylinder made of glass or plexiglass. Thin plastic or glass spacers separate the plane and the cylinder. The axis of the cylinder is horizontal, so that the thickness $\delta$ of the gap increases approximately quadratically with the distance $x$ from 
(a)

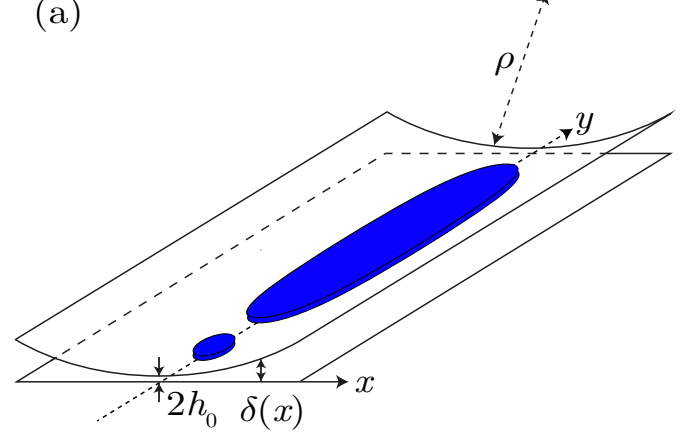

(b)

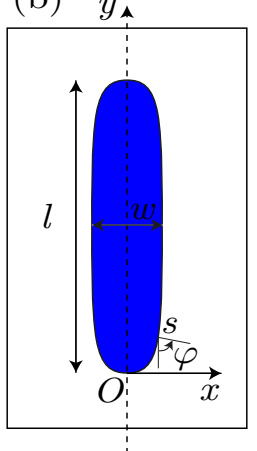

(c)

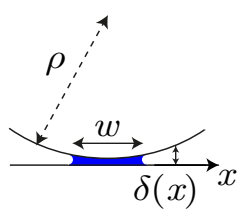

(d)

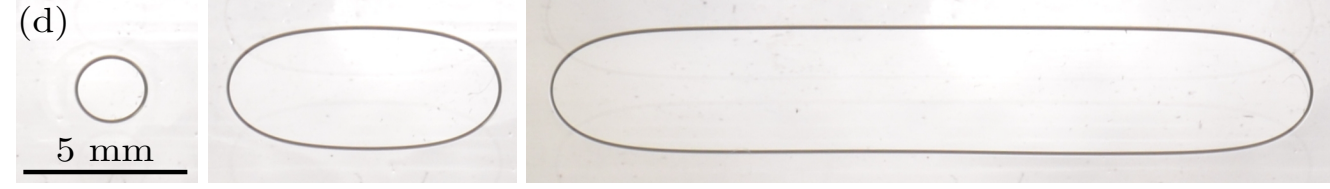

FiguRE 2. (a) Capillary bridges between a planar surface and a cylinder of radius $\rho$ with a horizontal axis. The gap between both walls at a distance $x$ from the line of closest gap is approximately $\delta(x)=2 h_{0}+x^{2} / 2 \rho$. (b) Top view, parametrization of the drop profile. The curvilinear coordinate $s$ is the distance to the extremity of the drop measured along its contour. The normal to the boundary in the horizontal plane makes an angle $\varphi$ with the $O y$ axis. $l$ is the length of the drop and $w$ its width, measured at the apparent contact line. (c) Side view of the capillary bridge. (d) Bottom view of drops of silicone oil trapped between a glass plate and a glass cylinder lying horizontally. The drops are seen from below, through the horizontal glass plane. The radius $\rho$ of the cylinder is $70 \mathrm{~mm}$ and the minimal gap $h_{0}=244 \mu \mathrm{m}$. Small droplets adopt a circular shape. As the volume of oil increases, drops are observed to stretch along the cylinder axis, where the gap is smallest. The drop width saturates to a finite value $w^{*} \simeq 3.4$ $\mathrm{mm}$.

the line of closest gap: $\delta(x) \simeq 2 h_{0}+x^{2} /(2 \rho)$, where $2 h_{0}$ is the minimal thickness and $\rho$ is the radius of curvature of the upper cylindrical wall. In our experiments, $h_{0}$ is of order $100 \mu \mathrm{m}$ while $\rho \sim 5 \mathrm{~cm}$. The gap thickness is small enough and the setup is horizontal so that gravity has a negligible effect on the shapes of drops. We introduce a liquid bridge of totally wetting silicone oil between the facing walls. The drop tends to get trapped in the region with the smallest gap, along the axis of the cylinder, where the solid/liquid contact area is largest for a given volume. We image these drops through the bottom glass plate from below using a digital SLR camera. As revealed by figure 2d, small droplets remain circular and form a puck, whereas larger liquid volumes stretch along the shallow region. The width $w$ of large drops saturates to an asymptotic value $w^{*}$ that depends on $\rho$ and $h_{0}$. We observe that $w^{*}$ is typically a few millimeters.

\subsection{Theoretical prediction of the shape}

In the absence of gravity, the interplay between the in-plane curvature and the height of the gap sets the shape of the drop. In a static situation, the pressure in the drop is constant, so that the position of the air-liquid interface is given by equation 2.11 with $\kappa$ being a constant that depends on the volume of the drop. The gap thickness is given by $\delta(x)=2 h_{0}+x^{2} / 2 \rho$, where $2 h_{0}$ is the minimal thickness and $\rho$ is the radius of curvature of the upper cylindrical wall. As shown in figure $2 \mathrm{~b}$, the distance to one extremity of the drop along the contour is the curvilinear coordinate $s$, and the local orientation of the interface in the plane of the plates is defined by the angle $\varphi$, so that the curvature of the projected shape in the $O x y$ plane is $1 / R=\mathrm{d} \varphi / \mathrm{d} s$. The shape of the drop in the plane 


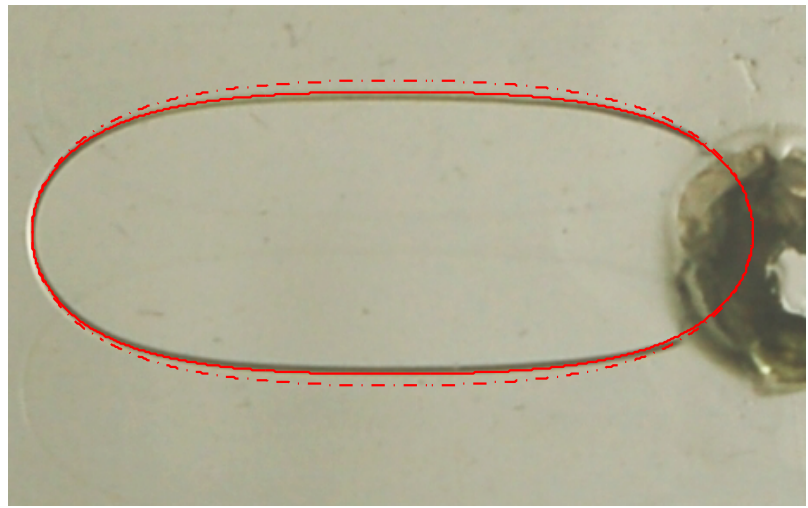

Figure 3. Top view of an elongated drop between a plane and a cylinder. The minimal gap and radius of the cylinder are $2 h_{0}=295 \mu \mathrm{m}$ and $\rho=70 \mathrm{~mm}$. We superimpose the experimental profile with the numerical solution of equation 3.3 (continuous line). Both are in excellent agreement. The dashed line is the profile computed using equation 3.4 neglecting the coupling between both terms in the curvature, i.e. assuming that the curvature of the interface would be $\kappa=2 / \delta(x)-1 / R$. The latter profile does not match the experimental one as well as the prediction of equation 3.3. The apparent thickness of the drop boundary is equal to the radius of curvature of the meniscus, which at first order is one half of the gap thickness $\delta$. The accuracy of the comparison with our model in not affected by the finite line thickness as the boundary appears sharp enough.

of the plates is thus given by

$$
\kappa=\frac{2}{\delta(x)}-\frac{\pi}{4} \frac{\mathrm{d} \varphi}{\mathrm{d} s}
$$

We take one extremity of the drop as a reference point: $\varphi=0, s=0$. Since $\kappa$ is constant, we have

$$
\frac{2}{\delta(x)}-\frac{\pi}{4} \frac{\mathrm{d} \varphi}{\mathrm{d} s}=\frac{1}{h_{0}}-\frac{\pi}{4} \frac{\mathrm{d} \varphi}{\mathrm{d} s}(s=0)
$$

or, noting that $\mathrm{d} \varphi / \mathrm{d} s(s=0)=\mathcal{C}_{0}$

$$
\frac{\mathrm{d} \varphi}{\mathrm{d} s}=\mathcal{C}_{0}+\frac{8}{\pi} \frac{1}{2 h_{0}+x^{2} / 2 \rho}-\frac{4}{\pi h_{0}}
$$

The choice of $\mathcal{C}_{0}$ then enables one to integrate 3.3. For given values of $h_{0}$ and $\rho$, the value of $\mathcal{C}_{0}$ sets the length or volume of the drop, and uniquely determines its shape. A similar approach was used by Paterson (1996) to solve the shape of a meniscus in a vertical Hele-Shaw cell of constant gap in which the deformations of interfaces are imposed by gravity instead of confinement gradients.

To compare the prediction of the model with the experimental shapes of the drops, we solve equation 3.3 numerically using MATLAB. We select the value of the curvature $\mathcal{C}_{0}$ such that the length of the computed profile matches that of the experiment. Figure 3 compares the experimentally measured boundary of an elongated drop with the prediction of the numerics, showing an excellent match of the shapes all along the drop boundary. If one neglects the coupling between the in-plane and transverse curvatures, assuming $\kappa=1 / h-1 / R$, equation 3.3 is changed to

$$
\frac{\mathrm{d} \varphi}{\mathrm{d} s}=\mathcal{C}_{0}+\frac{2}{2 h_{0}+x^{2} / 2 \rho}-\frac{1}{h_{0}}
$$

The numerical solution of equation 3.4 matching the experimentally measured drop 
length is also shown in figure 3. It does not match the experimental data as well and overestimates the width of elongated drops by approximately $8 \%$. As a comparison, the apparent thickness of the drop boundary due to the meniscus radius of curvature is at first order equal to one half of the gap thickness. As $\delta$ is typically only approximately $1 \%$ of the drop width, it does not perturb the accuracy of the comparison of the model and experiment.

\subsection{Width of large drops}

Large drops stretch along the region of smallest gap, and their width saturates to a value $w^{*}$, measured at the apparent contact line. We follow the derivation of section 2 to express $w^{*}$ as a function of $h_{0}$ and $\rho$. We consider the mechanical equilibrium of one half of a very long drop, and calculate the forces applied in the $y$ direction along the length of the drop. In the vicinity of the middle of the drop, the oil/air boundary in the plane of the plates is close to a straight line, so that the curvature of the interface is simply $2 / \delta^{*}$ where $\delta^{*}$ is the thickest gap explored by the drop. The pressure inside the drop is thus $\Delta P=2 \gamma / \delta^{*}$. The pressure force acting on one half of the drop is $\Delta P$ multiplied by the area of the cross-section of the drop in its central region:

$$
F_{P}=\frac{2 \gamma}{\delta^{*}}\left[2 \int_{0}^{\frac{w^{*}}{2}} \delta(x) \mathrm{d} x-\frac{\pi}{4} \delta^{* 2}\right]
$$

or

$$
F_{P}=\frac{2 \gamma}{2 h_{0}+\frac{w^{* 2}}{8 \rho}}\left[2 \int_{0}^{\frac{w^{*}}{2}}\left(2 h_{0}+\frac{x^{2}}{2 \rho}\right) \mathrm{d} x-\frac{\pi}{4}\left(2 h_{0}+\frac{w^{* 2}}{8 \rho}\right)^{2}\right]
$$

Pressure forces are balanced by surface tension. As derived in section 2 above, the projected length of the lines normal to $O y$ where surface tension acts involves two terms. The projection along $O x$ of the lines where the drop reaches the solid has length $2 w^{*}$. The length of the menisci on the side of the drop is $\pi \delta^{*}$. The resulting capillary force is thus

$$
F_{\gamma}=\gamma\left(2 w^{*}-\pi\left(2 h_{0}+\frac{w^{* 2}}{8 \rho}\right)\right)
$$

The balance of capillary and pressure forces now reads

$$
2 w^{*}-2 \pi h_{0}-\pi \frac{w^{* 2}}{8 \rho}=\frac{2}{2 h_{0}+\frac{w^{* 2}}{8 \rho}}\left[2 h_{0} w^{*}+\frac{w^{* 3}}{24 \rho}-\pi h_{0}^{2}-\frac{\pi w^{* 4}}{256 \rho^{2}}-\frac{\pi w^{* 2} h_{0}}{8 \rho}\right]
$$

Using the fact that $\rho \gg w^{*} \gg h_{0}$ and keeping the largest terms gives the asymptotic width $w^{*}$ of elongated drops:

$$
w^{*}=\left(12 \pi h_{0}^{2} \rho\right)^{1 / 3}
$$

The saturation of $w$ also implies the existence of an asymptotic value $\mathcal{C}_{0}^{*}$ of the extremal in-plane curvature $\mathcal{C}_{0}$ for large drops. Using 3.3 we obtain:

$$
\mathcal{C}_{0}^{*}=\frac{4}{\pi}\left(\frac{1}{h_{0}}-\frac{2}{2 h_{0}+\frac{w^{* 2}}{8 \rho}}\right)
$$

Combining with the result of equation 3.9, and in the limit $h_{0} \ll \rho$, one finds the minimal value of $\mathcal{C}_{0}$ for a given geometry:

$$
\mathcal{C}_{0}^{*} \simeq\left(\frac{9}{4 \pi h_{0}^{2} \rho}\right)^{1 / 3}
$$


so that

$$
w^{*} \mathcal{C}_{0}^{*}=3
$$

The scaling results in equations 3.9 and 3.11 may also be derived in a simpler way, invoking the constant interfacial curvature. In the central part of long drops, the gap thickness is $\delta^{*}=2 h_{0}+w^{* 2} / 8 \rho$, and the curvature of the interface is $\kappa^{*}=2 / \delta^{*}$. The observed orders of magnitude for $h_{0}, \rho$ and $w^{*}$ are such that $h_{0} \gg w^{* 2} / \rho$, so that

$$
\kappa^{*} \simeq \frac{1}{h_{0}}-\frac{w^{* 2}}{16 \rho h_{0}^{2}}
$$

At the end caps, the curvature of the drop is

$$
\kappa^{*}=\frac{1}{h_{0}}-\mathcal{C}_{0}^{*}
$$

Assuming that there is only one characteristic length scale $w^{*}$ to describe the in-plane shape of drops, one necessarily has $\mathcal{C}_{0}^{*} w^{*} \sim 1$. Using this last result and combining equations 3.13 and 3.14 , one indeed obtains:

$$
w^{*} \sim\left(h_{0}^{2} \rho\right)^{1 / 3}
$$

Figure 4a compares the measured value of the width of long drops with the theoretical result of equation 3.9. Both experimental $\left(w_{\text {exp }}^{*}\right)$ and numerical $\left(w_{\text {num }}^{*}\right)$ results are in close agreement with the prediction $w^{*}$. As tested on a few numerical cases, if one neglects the coupling of in-plane and transverse interfacial curvatures, one overestimates the asymptotic drop width by approximately $8 \%$, which does not describe the experimental results as well. The numerically determined asymptotic value $\mathcal{C}_{0}^{*}$ num of the curvature at one extremity of long drops is also in excellent agreement with the theoretical expression of $\mathcal{C}_{0}^{*}$.

Using the result of equation 3.9, we define the dimensionless width and length of drops, $W=w / w^{*}$ and $L=l / w^{*}$. Plotting $W$ as a function of $L$ in figure $4 \mathrm{~b}$, we see that numerical and experimental data for various values of $h_{0}$ and $\rho$ collapse onto a single master curve. Small drops $(W, L \ll 1)$ do not explore the gradient of confinement and remain trapped in a region of almost constant gap: $\delta \simeq 2 h_{0}$. As a consequence, their shape is close to circular: $W \simeq L$. Conversely, under the effect of inhomogeneous confinement, larger drops stretch along the axis of the cylindrical wall: $L \rightarrow+\infty, W \rightarrow 1$.

\section{Conclusion}

We have described experimentally and theoretically the shapes of drops trapped between two inhomogeneously spaced walls, a plane and a horizontal cylinder. A simple model predicts accurately the contours of these menisci. We have shown that the shape of such capillary bridges between closely spaced walls is indeed sensitive to the correction given by Park \& Homsy (1984). The correct description of other experimental results on two-phase flows in Hele-Shaw geometries involves the coupling of in-plane and transverse interfacial curvatures. Many dynamical situations with a confined moving curved front should take this effect into account. This is, for instance, the case for all problems related to the Saffman and Taylor viscous fingering instability (Saffman \& Taylor 1958). Limat (1992), for instance, studied the dynamics of a moving front in a Hele-Shaw cell perturbed by an isolated wettability defect. More recently, Al-Housseiny et al. (2012) have described the modified fingering instability in Hele-Shaw cells with a confinement gradient along the flow direction. Anjos \& Miranda (2014) have proposed a theoretical 

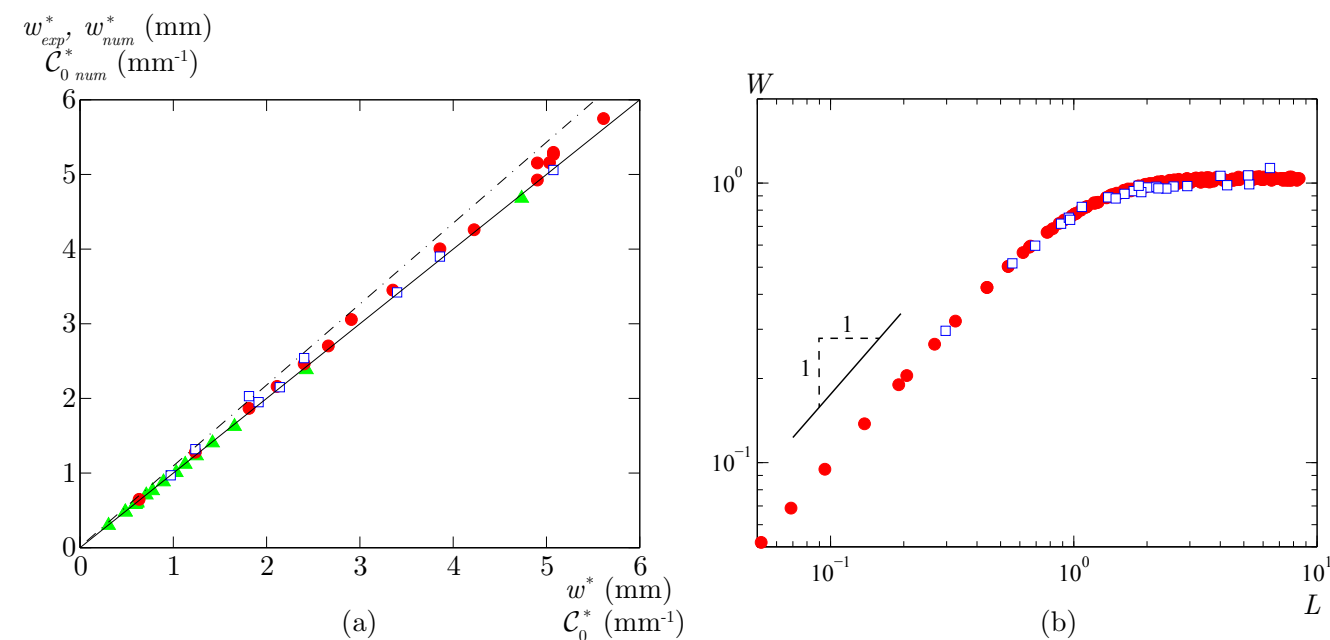

Figure 4. (a) Experimental $\left(w_{\text {exp }}^{*}, \square\right)$ and numerical $\left(w_{\text {num }}^{*}, \bullet\right)$ values of the asymptotic width of elongated drops plotted versus the theoretical prediction $w^{*}$ of equation 3.9. The equation of the continuous line is $w_{e x p}^{*}=w^{*}$. The equation of the dash-dotted line is $w_{\text {num }}^{*}=1.084 w^{*}$, as numerically computed using equation 3.4: this prediction slightly overestimates our experimental results. We also plot the numerical asymptotic value of the minimal in-plane curvature $\left(\mathcal{C}_{0 \text { num }}^{*}\right.$, $\Delta$ ) versus the theoretical prediction $\mathcal{C}_{0}^{*}$ of equation 3.11. (b) Dimensionless width $W=w / w^{*}$ of drops as a function of their dimensionless length $L=l / w^{*}$. The numerical results are shown as full dots $(\bullet)$ while the experimental results are displayed as open squares $(\square)$. Values of $h_{0}$ range from 30 to $500 \mu \mathrm{m}$ while $20<\rho<70 \mathrm{~mm}$. Small drops are circular $(W \simeq L)$ while large drops $(L \gg 1)$ elongate and their width saturates to the asymptotic limit $W=1$. The slope of the full line corresponds to a linear variation of $W$ with $L$.

description of the fingering instability in a cell whose thickness may evolve in time. In these dynamical situations, it is however likely that observation of the correction of Park \& Homsy (1984) experimentally will be challenging, as the behavior of moving contact lines is itself not very well understood.

I would like to warmly thank George M. Homsy for his kind advice and suggestions on this project, and Laurent Limat for interesting discussions and his enthusiastic encouragement. This research has been funded by the Interuniversity Attraction Poles Programme (IAP 7/38 MicroMAST) initiated by the Belgian Science Policy Office.

\section{Appendix}

In section 2, we give the first terms of the development of the curvature $\kappa$ of the surface of a drop of totally wetting liquid connecting two parallel plates separated by a gap of thickness $2 h$. In this appendix, following the work of Melrose (1966), we give the main steps leading to this result and enabling the computation of terms of any order. Melrose (1966) shows that the mean curvature $\kappa$ of an axisymmetric capillary bridge between two parallel planes is determined by the implicit equation

$$
h \kappa=1+\sqrt{c} K\left(-\frac{1}{c}\right)-\sqrt{c} E\left(-\frac{1}{c}\right)
$$

where $K$ and $E$ are complete elliptic integrals of the first and second kind, $c=R^{2} \kappa^{2}+2 R \kappa$ and $R$ is the radius of the drop at mid-height between the walls. We then write $h \kappa$ as a 
power series in $h / R$ :

$$
h \kappa=\sum_{n=0}^{+\infty} a_{n}\left(\frac{h}{R}\right)^{n}
$$

By developing the right-hand side of equation 4.1 and identifying the different orders in $h / R$, one can now compute iteratively the coefficients $a_{n}$ for arbitrary values of $n$. The terms up to order $(h / R)^{4}$ are given in section 2 .

\section{REFERENCES}

Ajaev, Vladimir S. \& Homsy, G.M. 2006 Modeling Shapes and Dynamics of Confined Bubbles. Annual Review of Fluid Mechanics 38 (1), 277-307.

Al-Housseiny, Talal T., Tsai, Peichun a. \& Stone, Howard a. 2012 Control of interfacial instabilities using flow geometry. Nature Physics 8 (10), 747-750.

Anjos, Pedro H. A. \& Miranda, José A. 2014 Influence of wetting on fingering patterns in lifting Hele-Shaw flows. Soft matter 10 (38), 7459-7467.

Bensimon, David, Kadanoff, Leo P., Liang, Shoudan, Shraiman, Boris I. \& Tang, Chao 1986 Viscous flows in two dimensions. Reviews of Modern Physics 58 (4), 977-999.

Bonn, Daniel, Eggers, Jens, Indekeu, Joseph, Meunier, Jacques \& Rolley, Etienne 2009 Wetting and spreading. Reviews of Modern Physics 81 (2), 739-805.

Butt, Hans-Jürgen \& Kappl, Michael 2009 Normal capillary forces. Advances in colloid and interface science 146 (1-2), 48-60.

Delaunay, C. 1841 Sur la surface de révolution dont la courbure moyenne est constante. Journal de Mathématiques Pures et Appliquées 6, 309-314.

Eichelsdoerfer, Daniel J, Brown, Keith a \& Mirkin, Chad a 2014 Capillary bridge rupture in dip-pen nanolithography. Soft matter 10 (30), 5603-5608.

Federle, Walter, Riehle, Mathis, Curtis, Adam S. G. \& Full, Robert J. 2002 An Integrative Study of Insect Adhesion: Mechanics and Wet Adhesion of Pretarsal Pads in Ants. Integrative and Comparative Biology 42, 1100-1106.

De Gennes, Pierre-Gilles 1985 Wetting: statics and dynamics. Reviews of Modern Physics 57 (3), 827-863.

de Gennes, Pierre-Gilles, Brochard-Wyart, Françoise \& Quéré, David 2004 Capillarity and wetting phenomena: drops, bubbles, pearls and waves. New York: Springer.

Geoffroy, S., Plouraboué, F., Prat, M. \& Amyot, O. 2006 Quasi-static liquid-air drainage in narrow channels with variations in the gap 294, 165-175.

Gondret, P. \& RABAud, M. 1997 Shear instability of two-fluid parallel flow in a Hele-Shaw cell. Physics of Fluids 9 (11), 3267-3274.

Limat, LAURENT 1992 Imperfect Hele-Shaw cell: a model for statics and dynamics of the interface perturbed by an isolated wettability defect. Comptes rendus de l'Académie des sciences. Série 2, Mécanique, Physique, Chimie, Sciences de l'univers, Sciences de la Terre 314, 1011-1016.

Melrose, James C. 1966 Model Calculations for Capillary Condensation. AIChE Journal 12 (5), 986-994.

Mitarai, Namiko \& Nori, Franco 2006 Wet granular materials. Advances in Physics 55 (1$2), 1-45$.

Nagel, Mathias, Brun, P.-T. \& Gallaire, François 2014 A numerical study of droplet trapping in microfluidic devices. Physics of Fluids 26 (3), 032002.

Park, C.-W. \& Homsy, G. M. 1984 Two-phase displacement in Hele Shaw cells: theory. Journal of Fluid Mechanics 139, 291-308.

PAterson, A. 1996 Mouillage de surfaces hétérogènes : cellule de Hele-Shaw imparfaite. Annales de Physique 21 (4), 337-436.

Paterson, A., Fermigier, M., P., Jenffer \& Limat, L. 1995 Wetting on heterogeneous surfaces: Experiments in an imperfect Hele-Shaw cell. Physical Review E 51 (2), 12911298.

Plouraboué, F. \& Hinch, E. J. 2002 Kelvin-Helmholtz instability in a Hele-Shaw cell. Physics of Fluids 14 (3), 922-929. 
Rabaud, Marc 1994 Dynamiques interfaciales dans l'instabilité de l'imprimeur. Annales de Physique, France 19, 659-690.

Rabaud, M., Couder, Y. \& Michalland, S. 1991 Wavelength selection and transients in the one-dimensional array of cells of the printer's instability. European Journal of Mechanics B/Fluids 10, 253-260.

Roman, B \& BICO, J 2010 Elasto-capillarity: deforming an elastic structure with a liquid droplet. Journal of physics. Condensed matter 22, 493101.

Saffman, P. G. \& Taylor, Geoffrey 1958 The penetration of a fluid into a porous medium or Hele-Shaw cell containing a more viscous liquid. Proceedings of the Royal Society A: Mathematical and Physical Sciences 245 (1242), 312-329.

Sauvage, P., Argentina, M., Drappier, J., Senden, T., Siméon, J. \& Di Meglio, J.M. 2011 An elasto-hydrodynamical model of friction for the locomotion of Caenorhabditis elegans. Journal of Biomechanics 44, 1117-1122. 